\title{
PROCESOS EMPRENDEDORES E INNOVADORES, COMO RESULTADO DE LA GESTIÓN DEL CONOCIMIENTO
}

\section{ENTREPRENEURIAL AND INNOVATIVE PROCESSES AS A RESULT OF KNOWLEDGE MANAGEMENT}

\section{* Garnica Roman, Derlys ${ }^{1}$.}

\author{
${ }^{1}$ Administradora de Empresas. Candidata a Magister en Gestión de la Innovación. Grupo de investigación CIPTEC- \\ Facultad de Ingenierías - Fundación Universitaria Tecnológico Comfenalco Cartagena, Cartagena de Indias - \\ Colombia. . *Correo electrónico: derlysgarnica@gmail.com
}

Recibido 21 de octubre 2016; aceptado 30 de marzo de 2017.

\section{RESUMEN}

El conocimiento es el activo intangible con mayor valor para las empresas, marcando una gran diferencia entre una y otra, transformándose en una gran ventaja competitiva y sostenible, brindando un valor agregado a las organizaciones si se gestiona en función de la sostenibilidad en el tiempo. Gestionar el conocimiento no es un proceso fácil, requiere de implementación de estrategias que generen acciones innovadoras orientadas a la diversificación empresarial, lo cual solo es posible si se da respuesta a diferentes 
Autor a quien dirigirse la correspondencia: *Garnica Román, Derlys Correo electrónico: derlysgarnica@gmail.com.

interrogantes inmersos en la planificación estratégica de las empresas.

Para tratar el conocimiento en las organizaciones, es importante partir de los sistemas educativos del país orientados a fomentar y desarrollar habilidades $y$ competencias en los procesos de formación; estos, en algunos casos se ven afectados por los cambios asociados a cada Gobierno en turno, los cuales proponen mejoras sustanciales las estrategias de mejora de los 4 pilares para el crecimiento (Ciencia, tecnología, educación, salud y familia).

Colombia, es un país con innumerables riquezas naturales y una sociedad emprendedora, que se podría transformar en un ejemplo de desarrollo; sin embargo al extrapolar la realidad se observa una sociedad castrada en crecimiento y desarrollo, impidiendo la dignificación del ser humano, evidenciándose principalmente por los pocos recursos para ciencia y tecnología; resaltando el sector educativo que cada día decrece producto de las políticas gubernamentales a espaldas de una realidad social cambiante, un sistema de salud que a voces reclama cambios sustanciales, y la familia que cada día se fractura más, bien sea por el crecimiento de familias disfuncionales o sencillamente se han cambiado lo roles de la tarea indelegable en la formación de los hijos.

Palabras Clave: Desarrollo, competencia, crecimiento, habilidad, emprendimiento, innovación, riqueza.

\section{ABSTRACT}

Knowledge is the intangible asset with greater value for companies, making a big difference between them, transforming into a great competitive and sustainable advantage, providing an added value to organizations if 
managed in terms of sustainability over time. Managing knowledge is not an easy process, it requires the implementation of strategies that generate innovative actions oriented to business diversification, which is only possible if you answer different questions immersed in the strategic planning of companies.

To deal with knowledge in organizations, it is important to start from the country's educational systems aimed at fostering and developing skills and competencies in training processes; These, in some cases, are affected by the changes associated with each government in turn, which propose substantial improvements to the strategies of improvement of the 4 pillars for Growth (science, technology, education, health and family).

Colombia, is a country with innumerable natural riches and an enterprising society, which could be transformed into an example of development; However to extrapolate the reality is observed a society castrated in growth and development, preventing the dignity of the human being, demonstrating mainly by the few resources for science and technology; Highlighting the educational sector that every day decreases the product of government policies behind a changing social reality, a health system that voices calls for substantial changes, and the family that each day is fractured more, either by the growth of dysfunctional families or simply have changed the roles of the undelegated task in the formation of children.

Keywords: Development, competition, growth, skill, entrepreneurship, innovation, wealth. 


\section{INTRODUCCION}

Los procesos emprendedores e innovadores son la base del crecimiento y desarrollo de los pueblos, considerándose como la base de la generación de riqueza en los entornos productivos de toda comunidad, jalonar o apalancar los procesos, es responsabilidad de todos (Audretsch \& Keilbach, 2004a; Audretsch \& Keilbach, 2004b, Audretsch \& Keilbach 2005; Audretsch, 2007).

Desde los procesos de formación se puede garantizar la base inicial de la generación de conocimiento, solo así se pueden propiciar espacios que faciliten procesos emprendedores e innovadores; fundamentados en el conocimiento individual, y su asocio en trabajo en equipo (Galindo \& Méndez, 2011). Solo se puede generar emprendimientos e innovaciones con el conocimiento propio de estos procesos, para ello es necesario desarrollar habilidades y competencias facilitadoras. El emprendimiento se concibe, como un conjunto de competencias, que parte de la actitud de la persona, para captar oportunidades de su entorno lo cual requiere de un pensamiento sistémico, convertirlas en ideas innovadoras con base en su creatividad y generar proyectos productivos en diferentes campos, para beneficio propio $y$ de su entorno (Uribe 2013). Aun, sin embargo, en algunas regiones de Colombia, y aquellos países en vías de desarrollo, no existen políticas claras inmersas en los planes de gobierno que dinamicen y fortalezcan las acciones de los entornos productivos (Galindo et al., 2012).

En el transcurrir del tiempo, han surgido muchos interrogantes en cuanto a orientaciones y características de los avances en el tema de emprendimiento e innovación, y estrategias utilizadas para su fomento y aplicación, aun, sin embargo, este es considerado como una nueva área del conocimiento que poco a poco cobra mayor importancia, tanto a nivel académico, como a nivel de los programas de los gobiernos (Galindo \& Méndez, 2011). Cabe anotar, que, en estos procesos cambiantes, la innovación ha surgido, con una herramienta de enfoque revolucionario que induce a resolver las problemáticas de las comunidades de manera diferente, generando valor en cada una de las posibilidades que mejoran las condiciones y calidad de vida de las comunidades (Galindo et al., 2012).

La generación de conocimiento, se ha convertido en la punta del iceberg, el cual es el foco principal de todo proceso de cambio, para ello las nuevas estrategias, están propiciando entornos productivos $y$ 
@LIMENTECH CIENCIA Y TECNOLOGÍA ALIMENTARIA ISSN 1692-7125. Volumen 15 No. 1, p. 86 - 96, año 2017 Facultad de Ingenierías y Arquitectura Universidad de Pamplona

competitivos orientados a la generación de ideas innovadoras y emprendedoras con ventajas competitivas que impulsan el aprendizaje de las personas estimulando en ellos avances sociales y económicos.

\section{GESTIÓN DEL CONOCIMIENTO.}

Según Ingrid Vania González Cisneros, la gestión del Conocimiento, se remonta desde los máximos exponentes de la filosofía griega. Platón (Atenas, 427 - 347 AC) quien afirmaba: "La existencia del mundo de ideas, invariables e invisibles sobre las que es posible adquirir un conocimiento certero"; a su vez indica que el conocimiento como disciplina se desarrolló de la forma de pensar de pioneros y académicos como Peter Drucker en la década de 1970, Karl-Erik Sveiby a finales de 1980, y Nonaka y Takeuchi en la década de 1990. Lo anterior se puede inferir que el conocimiento no es un término nuevo e improvisado, todo lo contrario, esta data desde las poblaciones antiguas, donde a pesar de no existir los grandes centros de estudios, el conocimiento fluía a partir de las experiencias de cada persona, y la información que pudiera manejar en el contexto donde se ubicara (Naranjo et al., 2016).

Nofal Nagles G, concibe la gestión del conocimiento como un proceso lógico, organizado y sistemático para producir, transferir y aplicar en situaciones concretas una combinación armónica de saberes. Con ello se puede concluir que el conocimiento es la base de todo proceso innovador, ya que este dota a las personas de herramientas que le permita cuestionarse ante situaciones diversas, analizar los entornos y por consiguiente facilitar la toma de decisiones, orientados a generar emprendimientos para la solución de problemas del entorno donde se desenvuelve; el conocimiento solo es válido y agrega valor, solo cuando se desarrollan habilidades y competencias que dan respuesta a cuestionamientos propios del ser humano frente a situaciones adversas (Consejo Nacional de Educación Superior, 2013).

Hoy en día, si se quiere ser competitivo, es necesario la utilización de manera eficiente todas las herramientas que otorga la gestión del conocimiento en las organizaciones, facilitar el aprendizaje en cada uno de sus miembros, acordes a los cambios presentados en el mercado globalizado, ya el éxito y posicionamiento en el mercado dependerá de la calidad de información, datos y herramientas con que cuente la organización (Chaparro, 2008; Chaparro, 2012).

María Fernanda Campo, Ministra de Educación Nacional (Colombia), afirma que 
@LIMENTECH CIENCIA Y TECNOLOGÍA ALIMENTARIA ISSN 1692-7125. Volumen 15 No. 1, p. 86 - 96, año 2017 Facultad de Ingenierías y Arquitectura Universidad de Pamplona

el desarrollo de un país depende de la oportunidad que tenga cada ciudadano para aprender y del espacio que se le dé para desarrollar a plenitud sus potenciales. De la satisfacción que cada quien alcance y del sentido que, a través de ese aprendizaje, le dé a su vida depende no solo su bienestar sino la prosperidad colectiva. Por esta razón el mundo entero se mueve en torno a la educación como uno de los factores clave para el progreso de las Naciones, en especial, aquellas en donde la pobreza y las brechas aún marcan una distancia de desigualdad entre sus ciudadanos. La sociedad de hoy requiere personas formadas para ejercer una ciudadanía responsable, que les permita desarrollarse integralmente como seres humanos; que les dé plena libertad para crear y participar de manera activa y significativa en las transformaciones de su entorno y que les brinde las herramientas para desempeñarse con eficiencia en un mundo caracterizado por nuevas y más complejas exigencias en materia laboral y productiva.

Es por ello que el desarrollo de habilidades y competencias propias de los requerimientos de los entornos productivos, se pueden lograr si se cuenta con una generación de conocimiento a partir del manejo de información, y datos como fuente de un conocimiento que agregue valor a los procesos (Chaparro, 2008; Chaparro, 2012).

\section{EMPRENDIMIENTO}

Emprendimiento, más que emprender es, generar cambios, cambios de actitud, en la manera de pensar y actuar, es generar riquezas a todo nivel, tanto emocional, espiritual, económica, mental, social. Desarrollando una mayor prevalencia en el conocimiento, cuando se genera sola y es consciente de las debilidades y capacidades propias y ajenas (Naranjo et al., 2016).

Aunque a la fecha no exista una definición global sobre emprendimiento, podemos empezar por algunas de las más reconocidas conceptualizaciones que se han dado al respecto. Emprendedor proviene de la palabra francesa "entreprende" que significa "llevar a cabo" y uno de los primeros en desarrollar conceptualizaciones al respecto fue Joseph Shumpeter (1978), para él la innovación y el cambio tecnológico se debían a la gente de espíritu libre y emprendedora, el veía a los emprendedores como agentes de cambio y desarrollo económico. También encontramos en la década de los 80's a Howard Stevenson quién interpretó la mentalidad emprendedora como un sistema de gestión apoyado más en las oportunidades que en los recursos, y finalmente citamos a la conocida escuela austriaca en donde varios autores difieren de los anteriores al definir el emprendimiento como un carácter oportunista del individuo u 
@LIMENTECH CIENCIA Y TECNOLOGÍA ALIMENTARIA ISSN 1692-7125. Volumen 15 No. 1, p. 86 - 96, año 2017 Facultad de Ingenierías y Arquitectura Universidad de Pamplona

organización respecto a los movimientos del mercado (Consejo Nacional de Educación Superior, 2013). Sin embargo, el emprendimiento, es citado como un área del conocimiento aun nueva en el marco global de la investigación (Chaparro, 2008; Chaparro, 2012), de igual forma en Colombia, la ley 1014 de enero 26 de 2006, define el emprendimiento como "Una manera de pensar y actuar orientada hacia la creación de riqueza. Es una forma de pensar, razonar y actuar centrada en las oportunidades, planteada con visión global y llevada a cabo mediante un liderazgo equilibrado y la gestión de un riesgo calculado, su resultado es la creación de valor que beneficia a la empresa, la economía y la sociedad"; aun sin embargo no se evidencian acciones, el Artículo $2^{\circ}$. Objeto de la Ley. La presente ley tiene por objeto: a) Promover el espíritu emprendedor en todos los estamentos educativos del país, en el cual se propenda y trabaje conjuntamente sobre los principios y valores que establece la Constitución y los establecidos en la presente ley. Si bien es cierto que algunas Instituciones cumplen con lo expreso en la ley, otras, no evidencian acciones que den cumplimiento a lo expreso en el objeto, el currículo no plantea estrategias para el fomento de la cultura emprendedora. La responsabilidad de sector educativo en ejercer seguimiento ha sido escasa, lo que en cierta manera se refleja en la poca aplicación y cumplimiento de lo inmediatamente expuesto en la Ley.

Siendo el emprendimiento un campo disciplinar, nuevo, en construcción teórica y conceptual; que requiere, para su implementación y generación de resultados, el establecimiento de políticas de gobierno, planes de gobierno, estrategias y programas sociales. Siendo así, (Castellano, 2012) infiere, que si se trata de la implementación de una política pública de emprendimiento se busca a mediano y largo plazo con acciones a corto plazo, una cultura emprendedora y empresarial, que cree unas nuevas condiciones de desarrollo económico social basado en el emprendimiento. Cabe anotar, que esto requiere la aplicación de enfoques que tendrá repercusión en todas las estructuras del conocimiento, donde cada persona comienza a construir su propio crecimiento, basado en el conocimiento logrado, bien sea para mejorar.

De acuerdo a la Revista Dinero Digital, en su artículo de Mayo 23 de 2017, Colombia tiene más potencial en emprendimiento que la UE, Norteamérica y Asia, es el potencial emprendedor de Colombia, equivalente a la capacidad y el interés de la población de crear su propio negocio, es más alto que el promedio regional y global de acuerdo a una investigación de la Universidad de Múnich 
@LIMENTECH CIENCIA Y TECNOLOGÍA ALIMENTARIA ISSN 1692-7125. Volumen 15 No. 1, p. 86 - 96, año 2017 Facultad de Ingenierías y Arquitectura Universidad de Pamplona

presentada hoy. El informe Global Entrepreneurship Monitor AGER 2016 explica que el potencial emprendedor de la población colombiana es del $80 \%$, mientras que el promedio latinoamericano es del $71 \%$ y el mundial del $43 \%$. En el caso de Norteamérica ese indicador el del $47 \%$, en Asia llega al 50 \% y en la Unión Europea el promedio es del $39 \%$, según lo reveló el informe elaborado por la Universidad de Múnich (Alemania) con el patrocinio de Amway. Justamente, el $52 \%$ de las personas menores a 35 años tienen un alto potencial para ser emprendedores, frente a un $33 \%$ de la población que tiene 50 años o más que también tiene capacidades para serlo. reto es lograr que esa porción de la población que tiene potencial pueda aprovecharlo, ya que debido a la falta de financiación $y$ confianza inversionista muchos proyectos se quedan en un archivo de Excel. El informe concluye, "En los próximos años el reto será transformar el emprendimiento en una forma de hacer negocios 'impulsada por la oportunidad' en lugar de basada en la necesidad'”.

Siendo así, las condiciones están dadas, para que el emprendimiento, más que una teoría, sea la mejor opción para que los pueblos emerjan del letargo económico y social en el que se encuentran, ya que desde los entornos productivos se perciben actitudes que propician la generación del conocimiento y el fortalecimiento y desarrollo de competencias propias de todo emprendedor (Castellano, 2012).

\section{INNOVACIÓN}

La innovación y emprendimiento, son nuevos conceptos que van de la mano hacia el desarrollo económico y social de las comunidades, siendo inclusiva todos, la innovación inclusiva requiere cambios institucionales a diferentes niveles para Favorecer los procesos de innovación. En la medida en que las sociedades presenten desigualdades tano económicas como políticas y sociales, como argumenta Berdegue (2005), los derechos de propiedad son sesgados (por ejemplo, los pobres no tienen acceso a tierras o créditos), la estratificación social bloquea la formación de redes sociales, y la corrupción y represión política.

Definir Innovación, podría centrarse en conceptos subjetivos de acuerdo a los interese de cada persona. Escorsa y Valls (1997) recogen algunas:

S. Gee (1981): “La innovación es el proceso mediante el cual, a partir de una idea, invención o reconocimiento de una necesidad, se desarrolla un producto, técnica o servicio útil." 
@LIMENTECH CIENCIA Y TECNOLOGÍA ALIMENTARIA ISSN 1692-7125. Volumen 15 No. 1, p. 86 - 96, año 2017 Facultad de Ingenierías y Arquitectura Universidad de Pamplona

J. Pavón y R. Goodman (1981): “Innovación es el conjunto de actividades, inscritas en un determinado período de tiempo y lugar, que conducen a la introducción con éxito en el mercado, por primera vez, de una idea en forma de nuevos o mejores productos, servicios o técnicas de gestión y organización" (Castellano, 2012).

P. F. Drucker (1986): “Innovación es el uso sistemático, como oportunidad, de los cambios en la sociedad, en la economía, en la demografía y en la tecnología”

\section{A. Piatier (1987): "La innovación es una idea transformada en algo vendido o usado".}

Cotec (1998): “La innovación es el complejo proceso que lleva las ideas al mercado en forma de nuevos o mejorados productos 0 servicios. Este proceso está compuesto por dos partes no necesariamente secuenciales y con frecuentes caminos de ida y vuelta entre ellas. Una está especializada en la creación del conocimiento y la otra se dedica fundamentalmente a su aplicación para convertirlo en un proceso un producto o un servicio que incorpore nuevas ventajas para el mercado".

Bajo estos conceptos se puede considerar que la innovación no es opcional, es esencial para el desarrollo y crecimiento de los pueblos, siendo claro que el emprendimiento e innovación actualmente forman parte de la vida cotidiana de cualquier sociedad desarrollada, siempre y cuando los procesos se gestión bajo las condiciones de conocimientos espontáneos logrados a partir de cuestionamientos, análisis y desarrollos de conceptos, datos e información.

\section{CONCLUSIÓN}

El desarrollo y crecimiento de los pueblos, solo podrá existir cuando las personas sean conscientes de sus responsabilidades como actores directos en las acciones propias en la generación de valor. Es importante, que ante todos los cambios que puedan presentarse, principalmente, aquellos emanados de las políticas estatales incluyan estrategias que fomenten y propicien escenarios hacia la generación del conocimiento que solo puede darse cuando existe un equilibrio entre la Ciencia y Tecnología, Educación, salud y familias; considerado como los pilares del crecimiento.

Solo si existe una sociedad con conocimiento, formada y educada, se puede pensar en la generación de soluciones a las problemáticas sociales, económicas y políticas del país; siendo así, si se cuenta con conocimientos básicos y críticos tales como: planificación, gestión fiscal, financiera, contabilidad, de marketing, calidad de servicio al cliente, de gestión del recurso 
humano y procesos; podrán desarrollarse

innovadoras y emprendedoras.

\section{REFERENCIAS BIBLIOGRAFIAS}

Audretsch B. Entrepreneurship capital and economic growth, Oxford Review of Economic Policy, 2007; 23(1): 63-78.

Audretsch DB. y Keilbach M. Does entrepreneurship capital matter?, Entre preneurship: Theory and Practice.2004a; 28(5): 419-429.

Audretsch DB. y Keilbach M. Entrepreneurship and regional growth: An Evolutionary interpretation, Journal of Evolutionary Economics, 2004b. 14(5):605-616.

Audretsch DB. y Keilbach M. Entrepreneurship capital and regional growth. Annals of Regional Science, 2005; 39(3): 457-469.

Castellano JE. La innovación en Colombia. ¿Qué reto nos espera? Revista Estrategia Organizacional. 2012; 85-91.

Chaparro F. 2008. Reflexiones sobre las organizaciones del conocimiento: aplicación de la teoría Sveby de la matriz de inteligencia organizacional (mio) a las universidades y a las empresas. Bogotá: Universidad del Rosario.
Chaparro F. 2012. Marco conceptual para el análisis de los procesos de gestión del conocimiento en la universidad. Bogotá: Universidad del Rosario.

Consejo Nacional de Educación Superior (CNES). 2013. Política marco de investigación, ciencia, tecnología e innovación en el sector de la educación terciaria, 2034. Disponible en http://www.dialogoeducacionsuperior.edu .co/1750/articles-.

Galindo MA y Méndez MT. La actividad emprendedora y competitividad: factores que inciden sobre los emprendedores, Papeles de Europa. 2011; 22:61-75

Galindo MA, Ribeiro D y Méndez MT. Innovación y crecimiento económico: Factores que estimulan la innovación. Cuadernos de Gestión, 2012; 12: 51-58.

Ley 1014 de enero 26 de 20016, Congreso de la Republica de Colombia. Enero 26 de 2006.

Naranjo S, González DL, Rodríguez J. The Challenge of Knowledge Management in Colombian Higher Education Institutions. 
FOLIOS • Segunda época. 2016; 44: 151-

164.

Revista Dinero. 2017. Colombia tiene más potencial en emprendimiento que la UE, Norteamérica y Asia. Disponible en http://www.dinero.com/emprendimiento/a rticulo/reporte-global-de-emprendimientoager-2016-resultados-colombia/245668.

Uribe M, J. Ediciones de la U, 1를 Edic. (2013) Emprendimiento y Empresarismo. Bogotá. 